\title{
Securing Web Service Compositions: Formalizing Authorization Policies Using Event Calculus
}

\author{
Mohsen Rouached and Claude Godart \\ LORIA-INRIA-UMR 7503 \\ BP 239, F-54506 Vandœuvre-les-Nancy Cedex, France \\ \{mohsen.rouached, claude.godart\}@loria.fr
}

\begin{abstract}
This paper presents a formal model for composing security policies dynamically to cope with changes in requirements or occurrences of events. We address one particular issue - that of authorization within a Web services composition. In particular, we propose a dynamic authorization model which allows for complex authorization policies whilst ensuring trust and privacy between the components services.
\end{abstract}

\section{Introduction}

Service Oriented Computing (SOC) is gaining prominence as the technology of choice for integrating applications in diverse and heterogeneous distributed environments. It is widely recognized that one of the barriers preventing widespread adoption of this technology is a lack of products that support non-functional features of applications, such as security, transactionality and reliability. Such properties are of utmost importance for Web service composition languages to keep their promises. Security is a challeging aspect of Web service composition that has not been so far deeply investigated despite its importance [2]3. For instance, a first challenge is the definition, the verification, and the enforcement of security policies as the complexity of composite Web services grows. To cope with this complexity, it is useful to design a conceptual model that gives a structured way to think about security policies. Another challenge is that non-functional concerns should be addressed by external specifications for a better separation of concerns and for more modular composition specification. For example, if we extend WSBPEL with new constructs for each non-functional concern of the composition, it would evolve into a very complex language, which in turn would limit its acceptance. Furthermore, mixing the specification of the core logic of the composition with specifications of security features and other non-functional concerns into one unit would make the composition specification too complex and hard to maintain and evolve.

In this paper, we propose to use a formalism based on the Event Calculus $(\mathcal{E C})$ [5] to specify authorization policies Web services compositions. $\mathcal{E C}$ is interesting because it supports the direct representation of events that are used in such policies, and the advantage of such a formalism is that it allows for having a common representation for different security models, every service having its own security model. 
In the rest of the paper, we introduce in Section 2 the notion of authorization in the context of Web services composition. In Section 3, we present how we specify the policies using the $\mathcal{E C}$, and how the consistency can be checked. Section 4 is dedicated to related works. Finally, Section 5 concludes the paper and outlines some future directions.

\section{Formalizing Authorization for Composite Web Services}

Service Oriented Architecture allows for considerably more complex interaction models than the classical client/server model, including symmetric peer-to-peer interactions where both parties want to check authorisations, or multi-party composed services where authorization is an issue for each component service. Therefore, an appropriate authorization framework is needed to smooth the flow of a transaction between multiple services whilst respecting the privacy of the data used. This is a complex task since each individual service may have its own authorization requirements

\subsection{Basic Notations and Definitions}

In this work, we use two booleans autho ${ }^{+}$and $a u t h o^{-}$to model positive and negative authorizations respectively. Therefore a positive authorization is denoted by autho $o^{+}(s, o, a)$, where $s, o$, and $a$ stand for subject, object, and action respectively. This authorization holds if the value of autho $o^{+}(s, o, a)$ equals true and does not hold otherwise. Similarly, autho- $(s, o, a)$ models a negative authorization. Positive and negative authorizations are used at the specification level to state who is or is not allowed to do what. As we will show, the use of signed (i.e positive/negative) authorizations gives more flexibility in handling authorization rules.

Given a Web service, we distinguish between two states according to its role in the request. The first one is given by $s_{s r c}$ to express that the service $s$ represent the source (who submit the request). The second type is denoted by $s_{\text {targ }}$ to precise that the service $s$ is the target (who receive the request). To summarize, a service is seen as a resource that is provided within the system, to which access is controlled. A service can also request other services and is actively involved in computation.

\subsection{Authorization Model}

To provide a formal specification of the authorization policies, we adapt a simple classical logic form of the $\mathcal{E C}$, whose ontology consists of (i) a set of time-points isomorphic to the non-negative integers, (ii) a set of time-varying properties called fluents, and (iii) a set of event types (or actions). The logic is correspondingly sorted, and includes the predicates Happens, Initiates, Terminates and HoldsAt, as well as some auxiliary predicates defined in terms of these. Happens $(a, t)$ indicates that event (or action) $a$ actually occurs at time-point $t$. Initiates $(a, f, t)$ (resp. Terminates $(a, f, t))$ means that if event $a$ were to occur 
at $t$ it would cause fluent $f$ to be true (resp. false) immediately afterwards. $\operatorname{Holds} A t(f, t)$ indicates that fluent $f$ is true at $t$.

To achieve a complete specification that supports formal reasoning in $\mathcal{E C}$, the following elements must be represented in the model.

- Separation between source services $\left(s_{s r c}\right)$ and target services $\left(s_{\text {targ }}\right)$ depending on the role of the service when performing or receiving the effect of an operation.

- Functions that can be used as parameters in the basic predicate symbols of $\mathcal{E C}$. We define these functions as events that may occur during the composition execution. Below, the introduced events are explained. In these formulas, $V_{p}$ represents the set of parameters values for the operations supported by services.

- operation $\left(s, \operatorname{Action}\left(V_{p}\right)\right)$ : used to denote the operations specified in a policy function or event (see below).

- requestAction $\left(s_{\text {src }}\right.$, operation $\left.\left(s_{\text {targ }}, \operatorname{Action}\left(V_{p}\right)\right)\right)$ : represents the event that occurs whenever a service source attempts to perform an operation on a target service. Therefore, this is the event that will trigger a permission (or denial) decision to be taken by the target service's access controller.

- doAction $\left(s_{\text {src }}\right.$, operation $\left.\left(s_{\text {targ }}, \operatorname{Action}\left(V_{p}\right)\right)\right)$ : represents the event of the action specified in the operation term being performed by the service $s_{s r c}$ on the service $s_{\text {targ }}$.

- rejectAction $\left(s_{\text {src }}\right.$, operation $\left.\left(s_{\text {targ }}, \operatorname{Action}\left(V_{p}\right)\right)\right)$ : the event that occurs after the enforcement decision to reject the request by a particular source service to perform an action is taken.

- $\operatorname{permit}\left(s_{\text {src }}\right.$, operation $\left.\left(s_{\text {targ }}, \operatorname{Action}\left(V_{p}\right)\right)\right)$ : represents the permission granted to a source service to perform the action defined in the operation on the target service.

- $\operatorname{deny}\left(s_{\text {src }}\right.$, operation $\left.\left(s_{\text {targ }}, \operatorname{Action}\left(V_{p}\right)\right)\right)$ : used to denote that the source service, $s_{s r c}$, is denied permission to perform that action on the target service $s_{\text {targ }}$.

- In addition to the described $\mathcal{E C}$ predicates, we add specific predicate symbols. Indeed, in our case many of the function definitions above contain the tuple $\left(s_{\text {src }}\right.$, operation $\left(s_{\text {targ }}\right.$, Action $\left.\left(V_{p}\right)\right)$. To check if the members of this tuple are consistent with the specification of the Web service composition, we define the isValidComp predicate. As such it must be used in any rule where functions with the tuple $\left(s_{\text {src }}\right.$, operation $\left(s_{\text {targ }}, \operatorname{Action}\left(V_{p}\right)\right)$ are involved.

Having specified these elements, it is now possible to explain how the various symbols defined above can be incorporated into rules that represent the different types of information required to specify authorization policies able to support Web service composition requirements in terms of security. The complete authorization enforcement model is illustrated in Figure 1 As shown, once the service source makes a request to perform an action on the service target, the target service's access controller processes it. To do this, the access controller evaluates the 
request by referring to the policy repository and the access control model. If the action is permitted, the access control model will proceed to do the requested action. Otherwise, if the action should be denied, the access control system will reject the action. We precise that the scheme is symmetric, i.e each of the two services could be target, source, or target and source at the same time. As shown in Figure 1,

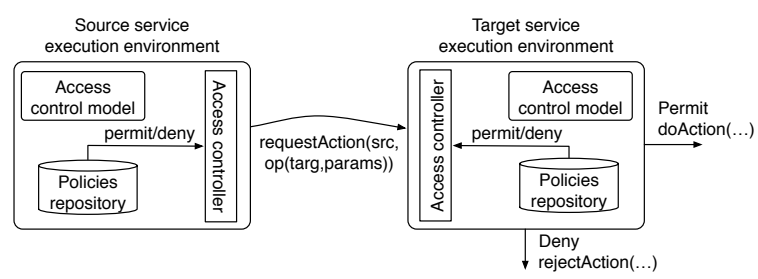

Fig. 1. Authorization Enforcement Model

we distinguish two scenarios to represent the enforcement model. The first scenario models the behaviour of the target service's access controller, generating a doAction event when an action is permitted. This event would trigger the relevant service behaviour rules thus causing the composition state to change according to the specification. The second one models a target service's access control monitor rejecting the action to prevent a denied operation from being performed.

\subsection{Authorization Specification}

In order to correctly interact with the enforcement model described above, each policy specification rule should initiate the appropriate policy function symbol (permit, deny) for each of the events. So for example, a positive authorization policy rule should specify that permit $\left(s_{s r c}, \operatorname{Operation}\left(s_{\text {targ }}, \operatorname{Action}\left(V_{p}\right)\right)\right)$ holds when the requestAction $\left(s_{\text {src }}\right.$, Operation $\left.\left(s_{\text {targ }}, \operatorname{Action}\left(V_{p}\right)\right)\right)$ event occurs and the constraints that control the applicability of the policy hold. Additionally, the fluent permit $\left(s_{s r c}\right.$, Operation $\left.\left(s_{\text {targ }}, \operatorname{Action}\left(V_{p}\right)\right)\right)$ should cease to hold once the action has been performed thus making it possible to re-evaluate the policy rule on subsequent requests to perform the action. The $\mathcal{E C}$ representation of this functionality is indicated in the $a u t o^{+}$specification shown in Figure 2, This also shows how each of the other policy types would be represented by rules in the formal notation. For each rule, the terms, $s_{s r c}, s_{\text {targ }}$, Action and Constraint, can be directly mapped to the source service, target service, action, constraint and event clauses used when specifying policies. The Constraint predicate is introduced to specify the pre- and post-conditions for each operation. It can be represented by a combination of HoldsAt terms.

The $a u t h o^{-}$specification shown in Figure2 represents a negative authorization policy by stating that, if the Constraint holds and the event requesting the action is performed happens, the action is denied. The second part of the rule shows how the deny fluent will be terminated once the decision to reject that action has been 


\begin{tabular}{|c|c|}
\hline Policy & Specification \\
\hline autho $^{+}$ & $\begin{array}{l}\text { Initiates }\left(\text { requestAction }\left(s_{\text {src }}, \text { operation }\left(s_{\text {targ }}, \operatorname{Action}\left(V_{p}\right)\right)\right), \text { permit }\left(s_{\text {src }},\right.\right. \\
\left.\text { operation }\left(s_{\text {targ }}, \operatorname{Action}\left(V_{p}\right)\right), t 1\right) \leftarrow \\
\text { isValidComp }\left(s_{s r c}, \text { operation }\left(s_{\text {targ }}, \operatorname{Action}\left(V_{p}\right)\right)\right) \wedge \text { Constraint } \\
\text { Terminates }\left(\operatorname{doAction}\left(s_{\text {src }}, \text { operation }\left(s_{\text {targ }}, \operatorname{Action}\left(V_{p}\right)\right)\right), \text { permit }\left(s_{\text {src }},\right.\right. \\
\left.\left.\text { operation }\left(s_{\text {targ }}, \operatorname{Action}\left(V_{p}\right)\right)\right), t 1\right) \leftarrow \\
\text { isValidComp }\left(s_{s r c}, \text { operation }\left(s_{\text {targ }}, \operatorname{Action}\left(V_{p}\right)\right)\right)\end{array}$ \\
\hline autho- & $\begin{array}{l}\text { Initiates }\left(\text { requestAction }\left(s_{\text {src }}, \text { operation }\left(s_{\text {targ }}, \operatorname{Action}\left(V_{p}\right)\right)\right), \operatorname{deny}\left(s_{\text {src }},\right.\right. \\
\left.\text { operation }\left(s_{\text {targ }}, \operatorname{Action}\left(V_{p}\right)\right), t 1\right) \leftarrow \\
\text { isValidComp }\left(s_{s r c}, \text { operation }\left(s_{\text {targ }}, \operatorname{Action}\left(V_{p}\right)\right)\right) \wedge \text { Constraint } \\
\text { Terminates }\left(\text { rejectAction }\left(s_{\text {src }}, \text { operation }\left(s_{\text {targ }}, \operatorname{Action}\left(V_{p}\right)\right)\right), \operatorname{deny}\left(s_{\text {src }},\right.\right. \\
\left.\left.\text { operation }\left(s_{\text {targ }}, \operatorname{Action}\left(V_{p}\right)\right)\right), t 1\right) \leftarrow \\
\text { isValidComp }\left(s_{s r c}, \text { operation }\left(s_{\text {targ }}, \operatorname{Action}\left(V_{p}\right)\right)\right)\end{array}$ \\
\hline
\end{tabular}

Fig. 2. Event Calculus Specification for Authorization Policies

taken, thus allowing the specification to be re-evaluated on subsequent requests. Note that the termination parts for these policies do not have any constraints and can be generically specified for the whole service composition.

\subsection{Conflicts}

In order to detect conflicts involving authorization policies, i.e. those that arise when it exists two policies defined for the same source, target and action: one being an authorization and the other one being a prohibition, we introduce the authConflict predicate that holds if an authorization conflict is detected. This predicate is defined as:

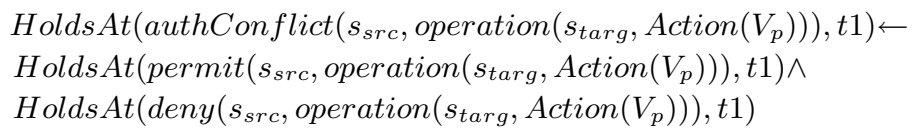

Let consider a typical example of authorization conflict, which arises when the same service is assigned to two roles that have opposite authorization permissions. To enable a complete specification of the different conflict cases that may arise, we introduce a further set of predicates, events, and fluents.

The additional predicates are Service(name), Action(name), Role(name), and ContradictoryRoles $(r 1, r 2, t, a)$. Service(name) denotes a service with a name name. Action (name) defines an action with a name name that a source can process on a target. Role(name) determines a role with the name name. ContradictoryRoles $(r 1, r 2, t, a)$ describes that roles $r 1$ and $r 2$ have opposite permissions for processing an action $a$ at $t$.

Then, the events introduced are AssignServiceRole $(s, r)$ that denotes a request of a service $s$ for assignment to a role $r$, RolePermitAction $(r, a)$ that specifies a request for permission of an action $a$ for a role $r$, and $\operatorname{RoleDeny\operatorname {Action}}(r, a)$ that defines a request for denial of action $a$ for a role $r$.

Finally, three fluents are specified: Assigned $(s, r)$ indicates that service $s$ is assigned to a role $r$, RoleHavePermission $(r, a)$ defines that a role $r$ is permitted 
to process action $a$, and AuthorizationConflict $(r 1, r 2)$ denotes that there is an authorization conflict in the composition (a service is assigned to contradictory roles).

Considering the elements described above, it is possible to define rules that can be used to recognise conflicting situations in the authorization policy specification. These rules are formalized as shown in Figure 3

\begin{tabular}{|c|c|}
\hline Rule & Specification \\
\hline $\mathrm{R} 1$ & $\begin{array}{l}\text { Initiates }(\text { RoleHavePermission }(r, a), \text { RolePermitAction }(r, a), t) \leftarrow \\
\text { Happens }(\text { RolePermitAction }(r, a), t) \wedge(\neg \text { HoldsAt }(\text { RoleHavePermission }(r, a) \\
, t))\end{array}$ \\
\hline R2 & $\begin{array}{l}\text { Terminates }(\text { RoleHavePermission }(r, a), \text { RoleDenyActivity }(r, a), t) \leftarrow \\
\text { Happens }(\text { RoleDenyActivity }(r, a), t) \wedge \text { HoldsAt }(\text { RoleHavePermission }(r, a), t)\end{array}$ \\
\hline R3 & $\begin{array}{l}\text { Initiates }(\text { Assigned }(s, r 1), \text { AssignU serRole }(s, r 1), t) \leftarrow \\
\text { Happens }(\text { AssignUserRole }(s, r 1), t) \wedge(\neg H \text { oldsAt }(\text { AuthorizationConflict }(r 1, \\
r 2), t))\end{array}$ \\
\hline $\mathrm{R} 4$ & $\begin{array}{l}\text { ContradictoryRoles }(r 1, r 2, t, a) \leftarrow(\text { HoldsAt }(\text { RoleHavePermission }(r 1, a), t) \wedge \\
(\neg \text { HoldsAt }(\text { RoleHavePermission }(r 2, a), t))) \mid(\text { HoldsAt }(\text { RoleHavePermission } \\
(r 2, a), t) \wedge(\neg \text { HoldsAt }(\text { RoleHavePermission }(r 1, a), t)))\end{array}$ \\
\hline R5 & $\begin{array}{l}\text { Happens }(\text { conflictEvent }, t) \wedge \text { Initiates }(\text { AuthorizationConflict }(r 1, r 2), \\
\text { conflictEvent }, t) \leftarrow H o l d s A t(\text { Authorized }(s, r 2), t) \wedge \text { Happens }(\text { Authorize - } \\
\text { Request }(r 1, s), t) \wedge \text { ContradictoryRoles }(r 1, r 2, a, t)\end{array}$ \\
\hline
\end{tabular}

Fig. 3. Rules for Authorization Conflicts

The first rule initiates the fluent RoleHavePermission $(r, a)$ when the event RolePermitAction $(r, a)$ happens if this fluent is currently not true. The second rule implements deny for role $r$ to process the action $a$ as a termination of fluent RoleHavePermission $(r, a)$ when RoleDenyActivity $(r, a)$ event happens. The third rule assigns service $s$ to the role $r$ when AssignU SerRole $(s, r)$ event happens if AuthorizationConflict $(r 1, r 2)$ between the role $r 1$ and some other role $r 2$ is not presented in the composition process. The fourth rule defines two roles, one of which has and another one does not have permission for some action. Here we note that we not fix which role has positive permission and which role has negative permission. Thus, ContradictoryRoles is symmetrical regarding $r 1$ and $r 2$. Finally, the fifth rule defines a notion of authorization conflict: the user requested the assignment for the second of two contradictory roles.

\section{Related Work}

There are few papers on security in the context of Web service compositions. We are aware only of the work presented in [4, which presents an access control framework for business processes in BPEL. Like our's, this framework is specific to the authorization problem. Our proposal is more formalized and it can be easily applicable to more security facets in Web service compositions (confidentiality, 
integrity, and authentication). In [7] the authors present a tool giving a simplified, business-policy-oriented view to its users, who are configuring secure Web services in their systems. They also based their proposal on WS-Security and WS-Policy but their tool does not support composite Web services.

In the project SECTINd1, a system architecture for local and global workflow system is proposed based on the XACML[6] and SAML. Security concerns are defined in OCL(Object Constraint Language) with model-driven UML tools. XACML is good for specifying policy in a specified domain. But it is not semantic rich enough for cross-organisational orchestration and high-level security requirements.

AO4BPEL 1 proposes an aspect-oriented extension to BPEL. It uses aspectsoriented concept to modularize cross-cutting concerns like security and performance in business processes. Although the AO4BPEL framework offers the modularity and dynamic adaptability to the Web service composition, it lacks semantic description of security aspects, business processes and business rules. This make conflicts detection and policy negotiation infeasible for securing the Web service composition.

\section{Conclusion}

In this paper, we presented a framework for managing authorization policies for Web service compositions. Specifically, we have described the use of Event Calculus and abductive reasoning for developing a language that supports specification and analysis of authorization policies for Web service composition. A complete implementation and an EC plug-in for Web service were developed and tested using test cases.

\section{References}

1. A. Charfi and M. Mezini. Aspect-oriented web service composition with ao4bpel. In $E C O W S$, volume 3250 of $L N C S$, pages 168-182. Springer, 2004.

2. D. Geer. Taking steps to secure web services. IEEE Computer, 36(10):14-16, 2003.

3. P. Hung, E. Ferrari, and B. Carminati. Towards standardized web services privacy technologies. In Proc of the IEEE International Conference on Web Services (ICWS'04), San Diego, CA, USA, July 2004.

4. H. Koshutanski and F. Massacci. An access control framework for business processes for web services. In XMLSEC '03: Proceedings of the 2003 ACM workshop on XML security, pages 15-24, New York, NY, USA, 2003. ACM Press.

5. R. Kowalski and M. J. Sergot. A logic-based calculus of events. New generation Computing 4(1), pages 67-95, 1986.

6. T. Moses. Extensible access control markup language (xacml) version 2.03 , Feb 2005.

7. M. Tatsubori, T. Imamura, and Y. Nakamura. Best-practice patterns and tool support for configuring secure web services messaging. In ICWS '04: Proceedings of the IEEE International Conference on Web Services (ICWS'04), page 244, Washington, DC, USA, 2004. IEEE Computer Society.

\footnotetext{
${ }^{1}$ http://qe-informatik.uibk.ac.at
} 